\title{
CFD study of an Electrical Submersible Pump (ESP) handling Two- Phase Liquid-Liquid flow
}

\author{
Deisy Becerra', Miguel Asuaje ${ }^{2}$, Nicolás Ratkovich ${ }^{1}$ \\ ${ }^{1}$ Department of Chemical Engineering, Universidad de los Andes \\ Carrera 1 \# 18a-12 Bogota, Colombia \\ ds.becerra10@uniandes.edu.co; n.rios262@uniandes.edu.co \\ ${ }^{2}$ Frontera Energy \\ Cll 100 \#9-25, Bogotá, Colombia \\ asuajem@fronteraenergy.ca
}

\section{Extended Abstract}

The Electrical Submersible Pump (ESP) is one of the most artificial lifting methods used in the last years, which consists of a serial arrangement of centrifugal pumps. One of the main concerns when handling crude oil is the formation of $\mathrm{O} / \mathrm{W}$ or W/O emulsions inside the pump, due to the shear rate imparted and the presence of high molecular weight substances that act as natural surfactants. Therefore, it is important to perform an analysis of the flow patterns inside the pump to increase the percentage of oil recovered using the centrifugal force and the difference in density between the oil and the water to generate the separation of liquid phases. For this study, a CFD model was developed on STAR-CCM+ software based on 3D geometry of a Franklin Electric 44004 " four-stage ESP. In this case, the modification of the last stage was carried out to improve the centrifugal effect inside the pump, and a perforated double tube was designed with three different holes configurations disposed at the outlet section, through which the cut water flows. The arrangement of holes used has different geometrical configurations such as circles, rectangles, and irregular shapes determined as grating around the tube. The twophase flow was modeled using an Eulerian approach with the VOF method, which predicts the distribution and movement of larger interfaces in immiscible phases. Different water-oil compositions were evaluated, such as 70-30\% v/v, 80-20\% v/v and $90-10 \% \mathrm{v} / \mathrm{v}$, respectively. Finally, greater recovery of oil was obtained. For the several compositions evaluated, the volumetric oil fraction was greater than 0.55 at the pump outlet. Similarly, it is possible to show an inversely proportional relationship between the WOR and the volumetric flow. The volumetric fractions evaluated, the oil flow increased approximately between $41 \%-10 \%$ for circular perforations and 49\%-19\% for rectangular shaped perforations, regarding the inlet flow. Besides, the elimination of the pump diffuser in the last stage of the pump reduced the head by approximately $20 \%$. 